\title{
Application of ANN and PCA to two-phase flow evaluation using radioisotopes
}

\author{
Robert Hanus ${ }^{1,}$, , Marcin Zych $^{2}$, Leszek Petryka ${ }^{3}$,Dariusz Świsulski ${ }^{4}$ and Anna Strzępowicz ${ }^{2}$ \\ ${ }^{1}$ Rzeszów University of Technology, Faculty of Electrical and Computer Engineering, 35-959 Rzeszów, Poland \\ ${ }^{2}$ AGH University of Science and Technology, Faculty of Geology, Geophysics and Environmental Protection, 30-059 Kraków, Poland \\ ${ }^{3}$ AGH University of Science and Technology, Faculty of Physics and Applied Computer Science, 30-059 Kraków, Poland \\ ${ }^{4}$ Gdańsk University of Technology, Faculty of Electrical and Control Engineering, 80-233 Gdańsk, Poland
}

\begin{abstract}
In the two-phase flow measurements a method involving the absorption of gamma radiation can be applied among others. Analysis of the signals from the scintillation probes can be used to determine the number of flow parameters and to recognize flow structure. Three types of flow regimes as plug, bubble, and transitional plug - bubble flows were considered in this work. The article shows how features of the signals in the time and frequency domain can be used to build the artificial neural network (ANN) to recognize the structure of the gas-liquid flow in a horizontal pipeline. In order to reduce the number of signal features the principal component analysis (PCA) was used. It was found that the reduction of signals features allows for building a network with better performance.
\end{abstract}

\section{Introduction}

Two-phase liquid-gas flow commonly occurs in nature and in industry, especially in: mining, nuclear, chemical, thermal and petrochemical engineering.

This type of flow may be studied using several methods, such as computer tomography, Coriolis flowmeters, optical equipment, PIV, LDA and nuclear techniques [1-8].

Knowledge of a two-phase flow structure is significant to properly conduct a number of industrial processes, so flow regime identification inspires many studies. Recent works show use of machine learning methods as e.g. artificial neural networks (ANN) [9-18] for this purpose. The flow structures classification with ANN is comprised of three steps: data acquisition and pre-processing, feature extraction, and structures classification. Generally, computational intelligence methods exploit various features of signals in the time, frequency and state-space domain.

The article shows how features of the signals from scintillation probe, analyzed in the time and frequency domain, can be used to build the ANN to recognize the structure of the gas-liquid flow in a horizontal pipeline. In order to reduce the number of signal features the principal component analysis (PCA) was used.

\section{Gamma-absorption method}

The gamma absorption technique is based on exponential decreasing of a gamma beam in function of composition and geometry of the absorbent [8]. The changes of the intensity of radiation are registered by the scintillation probes and converted into output electrical impulses [6].

The typical gamma-absorption set for two-phase gasliquid flow measurement is presented in Fig. 1.

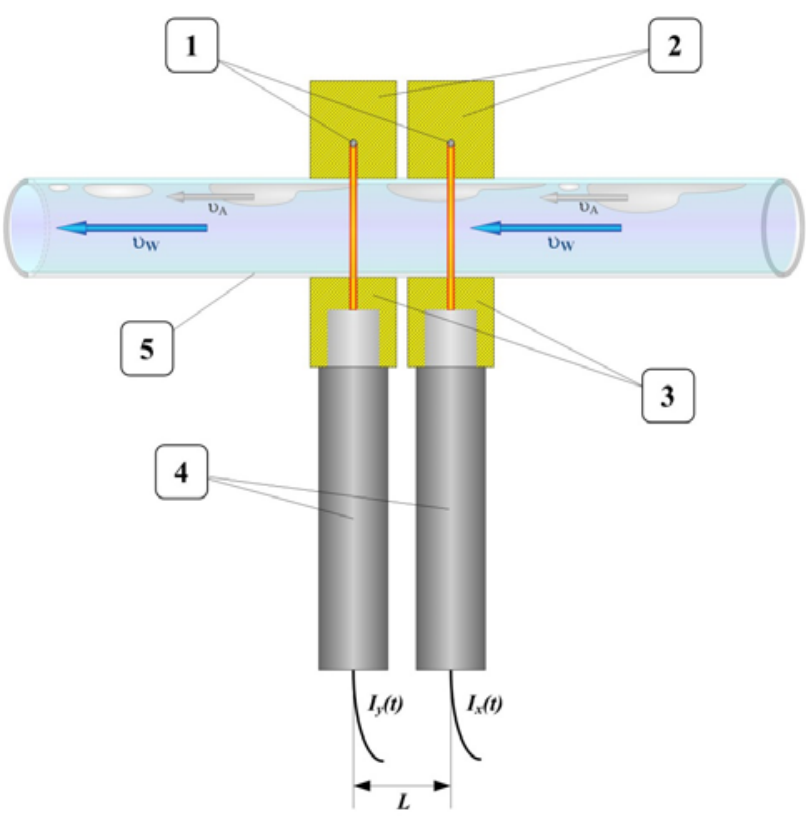

Fig. 1. The gamma-absorption measuring set: 1 - gamma radiation source, 2 - collimator of source, 3 - collimator of detector, 4 - scintillation probe, 5 - pipeline, $v_{A}, v_{W}$ - velocities of air and water respectively. [20]

Two sealed radioactive sources (1) emit gamma radiation beams with an energy of $59.5 \mathrm{keV}$ shaped by collimators (2). Photons pass through the pipeline with

\footnotetext{
Corresponding author: rohan@prz.edu.pl
} 
flowing compound (5) and detector's collimators (3) before achieving probes (4). Two sets consist of linear ${ }^{241} \mathrm{Am}$ sources with an activity of $100 \mathrm{mCi}$ and probes with 2" $\mathrm{NaI}(\mathrm{Tl})$ scintillation detectors [19]. For flow velocity measurement, the sets are placed at a distance of $L=97 \mathrm{~mm}$ between them [20]. Count signals $I_{x}(t)$ and $I_{y}(t)$ are recorded at the outputs of scintillation probes.

Measuring equipment described above was applied in the experimental set-up, built in Industrial Radiometry Laboratory of the AGH University of Science and Technology in Krakow, Poland (Fig. 2). Measurement data was collected in experiments carried out on a laboratory hydraulic installation with a horizontal pipe of $4.5 \mathrm{~m}$ length and inner diameter of $30 \mathrm{~mm}$. Detailed specification of experimental set-up presented in Fig. 2 is described in publications $[19,20]$.

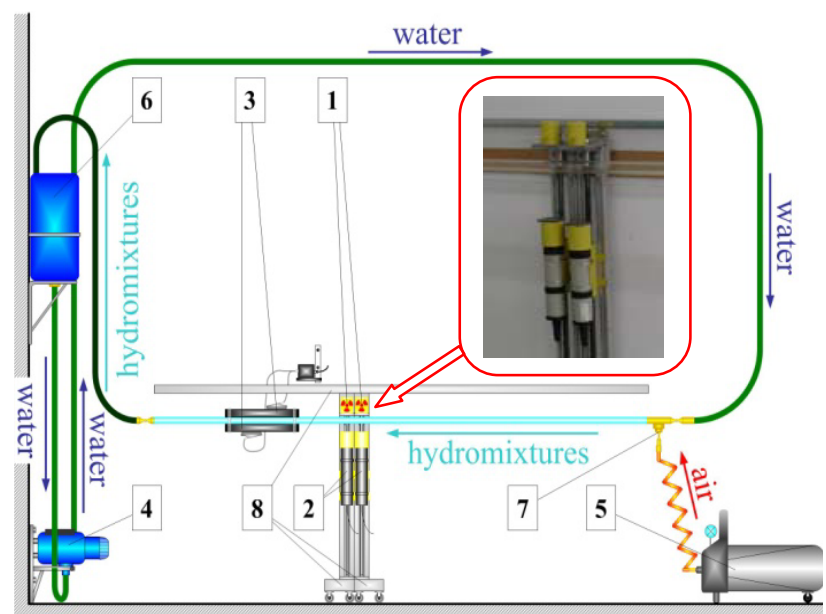

Fig. 2. Scheme of experimental set-up: 1 - gamma ray sealed source, 2 - scintillation probe, 3 - ultrasonic flow meter, 4 - pump, 5 - compressor, 6 - air-removing container, 7 - air nozzle, 8 - shifting system of gamma-absorption set. [20]

\section{Recorded signals}

In this work, three structures of air-water flow in horizontal pipeline are being considered: a plug flow, bubble flow, and transitional plug-bubble flow. For these structures of flow, the 300,000 sample signals were recorded in experiments denoted as: BUB6, BUB4, and BUB1 with a sampling rate of $1 \mathrm{kHz}$ [19]:

- BUB6 - plug flow: $v_{W}=0.90 \mathrm{~m} / \mathrm{s}, v_{A}=0.710$ $\mathrm{m} / \mathrm{s}$,

- BUB4 - transitional plug - bubble flow: $v_{W}=1.36$ $\mathrm{m} / \mathrm{s}, v_{A}=1.066 \mathrm{~m} / \mathrm{s}$,

- BUB1 - bubble flow: $v_{W}=1.92 \mathrm{~m} / \mathrm{s}, v_{A}=1.446$ $\mathrm{m} / \mathrm{s}$,

where $v_{W}$ - velocity of water was measured by ultrasonic flowmeter Uniflow 990. Velocity of gas phase $v_{A}$ was appointed using cross-correlation technique [2124].

Fig. 3 presents photos of particular types of flow structures and time records of the signals obtained in experiments BUB6, BUB4, and BUB1 [19]. a)
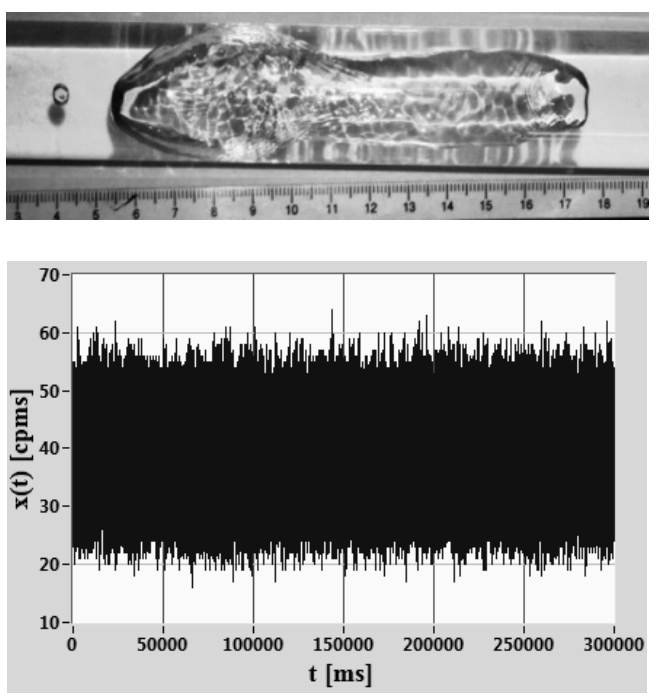

b)
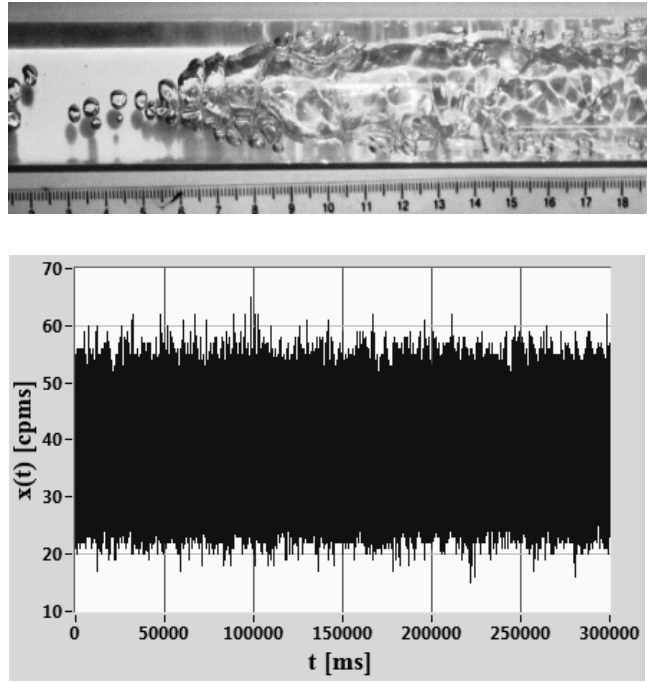

c)
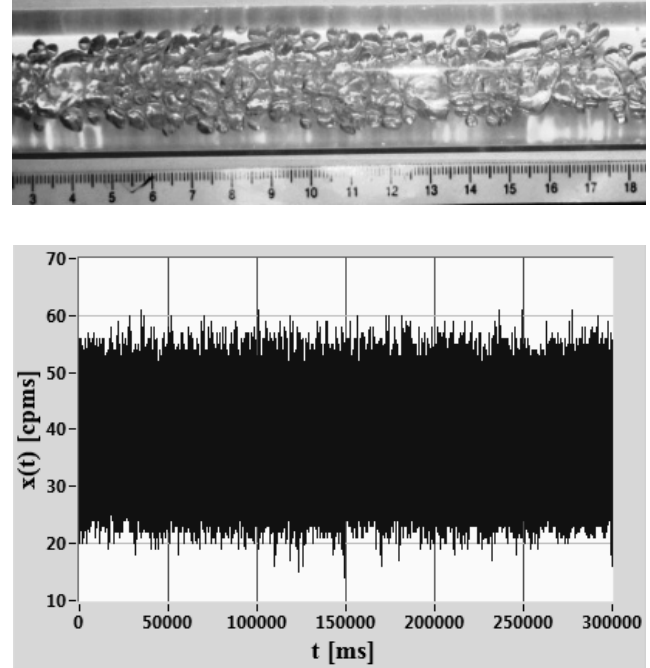

Fig. 3. Flow structures, and time records of signals obtained from scintillation probe in experiments (a) BUB6, (b) BUB 4 and (c) BUB1. [19] 


\section{Signals features extraction}

Based on the analysis of the data records registered in the experiments the following parameters (features) were calculated for the three types of the flow regimes (classes): "plug flow", "transitional plug - bubble flow", and "bubble flow":

a) in the time domain: mean value, variance, standard deviation, $4^{\text {th }}$ order moment, root mean square (RMS), skewness, kurtosis [19];

b) in the frequency domain: maximum value of the autospectral density function (ADF), maximum value of the cross-spectral density function (CSDF) magnitude, sum and relative sum of the ADF in the selected frequency range, sum and relative sum of the CSDF in the selected frequency range, the maximum value of the sum of selected amplitudes of Short-Time Fourier Transform (STFT) spectrogram, and area and relative area under the graph of STFT spectrogram sum in the selected frequency range [25].

A total of 16 features were extracted for data segments with a length of 10,000 samples and 50\% overlapping. The data analysis and computation of the features (predictors) were performed using LabVIEW software. Applied signal processing procedures are described in publications [19, 25].

\section{ANN and PCA application recognition of the flow regime}

In this work STATISTICA 12 Automated Neural Networks software was used for the construction of ANN [26]. Different artificial neural network structures were tested for obtaining the best one.

The best ANN configuration for total of 16 predictors proved to be Multi-Layer Perceptron (MLP) 16-8-3 (digits indicate the number of neurons in the input, hidden and output layer respectively). The number of samples for learning, testing and validation sets were 235 (about 70\%), 50 (about 15\%), 50 (about 15\%) respectively. Table 1 shows the specification and results of test for the obtained ANN. Tables 2-4 present results for learning, testing and validation respectively.

Table 1. Specification of MLP 16-8-3 ANN

\begin{tabular}{|c|c|}
\hline Parameter & Specification \\
\hline Quality (training) & $99.57 \%$ \\
\hline Quality (test) & $100 \%$ \\
\hline Quality (validation) & $100 \%$ \\
\hline Learning algorithm & BFGS 16 \\
\hline Activation function (hidden) & Tanh \\
\hline Activation function (output) & Linear \\
\hline
\end{tabular}

In order to reduce the number of predictors, the principal component analysis (PCA) was used. PCA explains the correlation structure of a set of predictors using a smaller set of linear combinations of them [27]. In general, the first principal component is the best summary of correlations among the predictors. This particular linear combination of the variables accounts for more variability than any other conceivable linear combination.

Table 2. Results for MLP 16-8-3 ANN (learning)

\begin{tabular}{|c|c|c|c|}
\hline & BUB1 & BUB4 & BUB6 \\
\hline Total & 76 & 80 & 79 \\
\hline Correct & 76 & 79 & 79 \\
\hline Incorrect & 0 & 1 & 0 \\
\hline Correct, \% & 100 & 98.75 & 100 \\
\hline Incorrect, \% & 0 & 1.25 & 0 \\
\hline
\end{tabular}

Table 3. Results for MLP 16-8-3 ANN (testing)

\begin{tabular}{|c|c|c|c|}
\hline & BUB1 & BUB4 & BUB6 \\
\hline Total & 16 & 18 & 16 \\
\hline Correct & 16 & 18 & 16 \\
\hline Incorrect & 0 & 0 & 0 \\
\hline Correct, \% & 100 & 100 & 100 \\
\hline Incorrect, \% & 0 & 0 & 0 \\
\hline
\end{tabular}

Table 4. Results for MLP 16-8-3 ANN (validation)

\begin{tabular}{|c|c|c|c|}
\hline & BUB1 & BUB4 & BUB6 \\
\hline Total & 20 & 13 & 17 \\
\hline Correct & 20 & 13 & 17 \\
\hline Incorrect & 0 & 0 & 0 \\
\hline Correct, \% & 100 & 100 & 100 \\
\hline Incorrect, \% & 0 & 0 & 0 \\
\hline
\end{tabular}

The second principal component is the second-best linear combination of the predictors, on the condition that it is orthogonal to the first principal component. The third component is the third-best linear combination of the predictors, on the condition that it is orthogonal to the first two components. The third component is derived from the variance remaining after the first two components have been extracted, and so on [27].

STATISTICA software was used for PCA after standardization of predictors [28]. The number of principal components was limited to four based on the scree plot (Fig. 4).

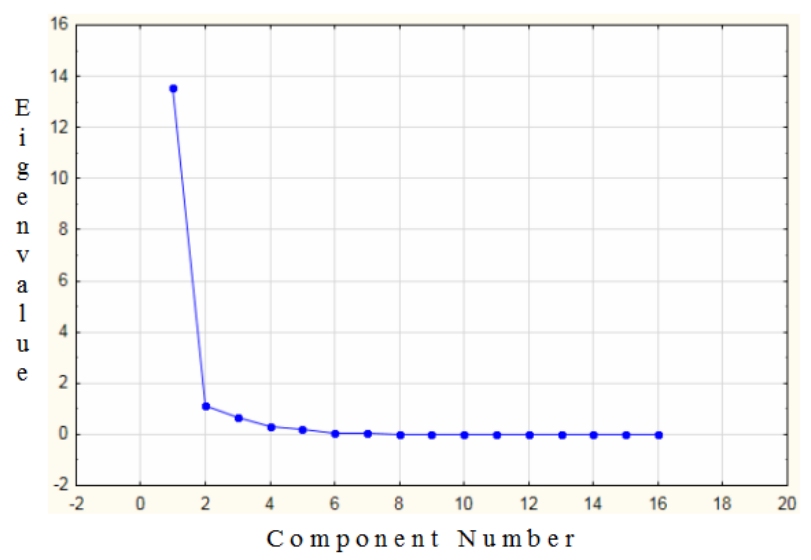

Fig. 4. Scree plot.

The selected principal components were used as predictors for ANN constructed using STATISTICA 
Automated Neural Networks software. The obtained specification and results for selected ANN MLP 4-4-3 with the highest performance are shown in Tables $5-8$. The type of flow structure was recognized with $100 \%$ accuracy using this ANN.

Table 5. Specification of MLP 4-4-3 ANN

\begin{tabular}{|c|c|}
\hline Parameter & Specification \\
\hline Quality (training) & $100 \%$ \\
\hline Quality (test) & $100 \%$ \\
\hline Quality (validation) & $100 \%$ \\
\hline Learning algorithm & BFGS 23 \\
\hline Activation function (hidden) & Exponential \\
\hline Activation function (output) & Softmax \\
\hline
\end{tabular}

Table 6. Results for MLP 4-4-3 ANN (learning)

\begin{tabular}{|c|c|c|c|}
\hline & BUB1 & BUB4 & BUB6 \\
\hline Total & 76 & 80 & 79 \\
\hline Correct & 76 & 80 & 79 \\
\hline Incorrect & 0 & 0 & 0 \\
\hline Correct, \% & 100 & 100 & 100 \\
\hline Incorrect, \% & 0 & 0 & 0 \\
\hline
\end{tabular}

Table 7. Results for MLP 4-4-3 ANN (testing)

\begin{tabular}{|c|c|c|c|}
\hline & BUB1 & BUB4 & BUB6 \\
\hline Total & 16 & 18 & 16 \\
\hline Correct & 16 & 18 & 16 \\
\hline Incorrect & 0 & 0 & 0 \\
\hline Correct, \% & 100 & 100 & 100 \\
\hline Incorrect, \% & 0 & 0 & 0 \\
\hline
\end{tabular}

Table 8. Results for MLP 4-4-3 ANN (validation)

\begin{tabular}{|c|c|c|c|}
\hline & BUB1 & BUB4 & BUB6 \\
\hline Total & 20 & 13 & 17 \\
\hline Correct & 20 & 13 & 17 \\
\hline Incorrect & 0 & 0 & 0 \\
\hline Correct, \% & 100 & 100 & 100 \\
\hline Incorrect, \% & 0 & 0 & 0 \\
\hline
\end{tabular}

\section{Conclusions}

In this paper an application of the gamma-absorption method in combination with ANN and PCA to recognize the gas-liquid flow regime in a horizontal pipeline has been presented.

A total of 16 features was extracted for data obtained from the gamma-absorption set based on ${ }^{241} \mathrm{Am}$ radioactive sources and $\mathrm{NaI}(\mathrm{Tl})$ scintillation probes. In order to reduce the number of predictors the principal component analysis was conducted. Next, four selected components were used as predictors for the construction of ANN.

It was found, that the reduction of predictors using PCA allows to build ANN with better performance (simpler structure and better accuracy) than without such a reduction.

\section{References}

1. G. Falcone, G.F. Hewitt, C. Alimonti, Multiphase flow metering: principles and applications (Elsevier, Amsterdam 2009)

2. M. Bottin, et al, Int. J. Multiphas. Flow 60, 161-179 (2014)

3. T. Xue, et al, Flow Meas. Instrum. 27, 29-36 (2012)

4. P. Vlasak, et al, J. Hydrol. Hydrom. 62, 241-247 (2014)

5. L. Petryka, M. Zych, R. Murzyn, Nukleonika 50, 43-46 (2005)

6. Z. Yu, B. Qincheng, H. Richa, Appl. Therm. Eng. 60, 398-410 (2013)

7. S.L. Soo (ed.), Instrumentation for fluid-particle flow (Noyes Publications, New Jersey, 1999)

8. W.A.S. Kumara, B.M. Halvorsen, M.C. Melaaen, Int. J. Multiphas. Flow 36, 467-480 (2010)

9. S. Blaney, H. Yeung, Flow Meas. Instrum. 19, 57 66 (2008)

10. O. Arjmandi-Tash, A. Zarghami, R. Zarghami, Particul. Sci. Technol. 32, 498-505 (2014)

11. Y. Chen, et al, Chem. Eng. Sci. 129, 156-167 (2015)

12. F. Johnsson, Int. J. Multiphas. Flow 26, 663-715 (2000)

13. C.M. Salgado, Prog. Nucl. Energy 52, 555-562 (2010)

14. Z. Sun, H. Zhang, Meas. Sci. Technol. 19, 015401 (2008)

15. G.H. Roshani, et al, Measurement 62, 25-32 (2015)

16. C.M. Salgado, Appl. Radiat. Isot. 67, 1812-1818 (2009)

17. B.M. Abbagoni, H. Yeung, Meas. Sci. Technol. 27, 084002 (2016)

18. M. Khorsandi, et al, Radiat. Meas. 59, 183-187 (2013)

19. R. Hanus, et al, EPJ $W o C$ 114, 02035 (2016)

20. R. Hanus, et al, EPJ WoC 92, 02021 (2015)

21. M.S. Beck, A. Plaskowski, Cross correlation flowmeters - their design and application (Adam Hilger, Bristol, 1987)

22. W.Q. Yang, M.S. Beck, Flow Meas. Instrum. 8, 7784 (1998)

23. L. Petryka, J. Oszajec, Nucl. Geoph. 7, 323-333 (1993)

24. J.S. Bendat, A.G. Piersol, Random data - analysis and measurement procedures (John Wiley, New York, 2010)

25. R. Hanus, et al, EPJ $W o C$ 114, 02036 (2016)

26. http://www.statsoft.com/Products/STATISTICA/ Automated-Neural-Networks

27. D.T. Larose, Data mining - methods and models (Wiley - Interscience, New Jersey, 2006)

28. http://www.statsoft.com/Portals/0/Products/PCA Analysis_with_STATISTICA.pdf 